Synthesis of polymer-based triglycine sulfate nanofibres by electrospinning

This article has been downloaded from IOPscience. Please scroll down to see the full text article.

2009 J. Phys. D: Appl. Phys. 42205403

(http://iopscience.iop.org/0022-3727/42/20/205403)

View the table of contents for this issue, or go to the journal homepage for more

Download details:

IP Address: 193.137.90.65

The article was downloaded on 11/11/2010 at 15:51

Please note that terms and conditions apply. 


\title{
Synthesis of polymer-based triglycine sulfate nanofibres by electrospinning
}

\author{
Dmitry Isakov $^{1}$, Albino M Martins ${ }^{2}$, Etelvina de Matos Gomes ${ }^{1}$, \\ Igor Bdikin $^{3}$, Ana Guimarães ${ }^{2}$, Tatjana Dekola ${ }^{1}$, Bernardo Almeida ${ }^{2}$, \\ Nuno M Neves ${ }^{2}$, Rui L Reis ${ }^{2}$ and Francisco Macedo ${ }^{1}$ \\ ${ }^{1}$ Physics Department, University of Minho, Campus de Gualtar, 4710-057 Braga, Portugal \\ 2 3B's Research Group-Biomaterials, Biodegradables and Biomimetics, Department of Polymer \\ Engineering, University of Minho, AvePark, Zona Industrial da Gandra, S.Cláudio do Barco, 4806-909 \\ Caldas das Taipas, Guimarães, Portugal \\ ${ }^{3}$ Department of Mechanical Engineering and TEMA, University of Aveiro, 3810-193 Aveiro, Portugal \\ E-mail: dmitry@ fisica.uminho.pt
}

Received 9 June 2009, in final form 21 July 2009

Published 23 September 2009

Online at stacks.iop.org/JPhysD/42/205403

\begin{abstract}
In this work we present the synthesis and characterization of polyethylene oxide (PEO) based triglycine sulfate $\left(\mathrm{NH}_{2}\left(\mathrm{CH}_{2} \mathrm{OOH}\right)_{3} \mathrm{H}_{2} \mathrm{SO}_{4}\right.$, TGS) nanofibres obtained by electrospinning. The fibres, with typical diameters of about $190-750 \mathrm{~nm}$ and above several hundred micrometres in length, present the nanocrystals of TGS embedded in a polymer matrix. The obtained nanofibres were characterized by FT-IR spectroscopy and the domain structure was examined by piezoforce microscopy. Dielectric permittivity measurements on the TGS-PEO nanofibres exhibit the characteristic ferroelectric-paraelectric phase transition at around $50{ }^{\circ} \mathrm{C}$.
\end{abstract}

(Some figures in this article are in colour only in the electronic version)

\section{Introduction}

In the last decade there has been an increased interest in the process of electrospinning for producing nanofibres due to its simplicity and low production cost $[1,2]$. The overwhelming majority of the published works are devoted to the application of polymer nanofibres on tissue engineering [3,4], biosensing [5,6], health care [7], membranes and filters [8]. However, electrospinning of nanofibres of organic/inorganic hybrids [9-11] and inorganic composite ceramic materials [12,13] has been generally limited to a description of the processing methods and structural characterization.

Hybrid organic/inorganic materials represent one of the most promising classes of one-dimensional materials. They can exhibit structural flexibility, high polarizability and nonlinear optical efficiencies, intrinsic to organic materials, and hydrophilic, mechanical and thermal stability characteristics of inorganic ones. These features can make semiorganic nanomaterials ideal for a new generation of multifunctional devices; among them the ferroelectrics play a significant role. Scaling down can be desirable in order to increase the sensitivity of materials due to an enhanced surface-tovolume ratio. In this context, the synthesis of ferroelectric nanofibres is very promising for intelligent device applications and can be used, for instance, in self-assembled nanoelectronic devices and memory cells with matrix addressing arrangement. Also, one-dimensional structures are good systems to aid in the understanding of nanoscale ferroelectricity and often exhibit novel properties when compared with their bulk counterparts [14-16].

Crystalline triglycine sulfate (TGS) is the best known semiorganic ferroelectric material and is widely used in infrared (IR) detection applications. The presence of ferroelectricity, its high pyroelectric and piezoelectric coefficients at ambient temperature, its low-cost and ease of fabrication make TGS a very attractive material both from the fundamental point of view as well as for applications. Despite its wide use in industry there are still many attempts to find new properties and applications of TGS at the microscopic level $[17,18]$. In fact, the reduction of thermal mass for improving the pyrodetecting ability of TGS was recently reported [19].

Many synthesis methods have been developed for the production of nanofibre materials with various structures. Among these methods, vapour-liquid-solid deposition [20], oxide-assisted growth [21], soft or hard template self-assembly 
synthesis [22,23] and the phase-separation method were used. However, nanomaterials produced by these conventional synthetic bottom-up methods are discontinuous objects, while electrospinning enables the production of continuous and uniform polymer nanofibres.

The physics and details of the electrospinning technique have been studied intensively and are described elsewhere $[24,25]$. In the conventional electrospinning process a high dc electric field is applied to a capillary tube loaded with a liquid polymer or a sol-gel precursor. At the end of the tube the droplet forms the shape of a Taylor cone, due to the competition between surface tension and the electrostatic force. When the applied voltage overcomes a threshold value, the electrostatic force becomes dominant and fibre jet emission begins. The emitted fibre jet experiences several instability modes where most of the fibre splitting, elongation and thinning are accomplished. Depending on the experimental conditions, diameters can be controlled down to tens of nanometres. In this work we present a method for producing polymerbased triglycine sulfate (TGS-PEO) nanofibres, composed of nanosized TGS grains dispersed in the polyethylene oxide (PEO) matrix.

\section{Experimental}

The TGS-PEO nanofibres were processed by the electrospinning method. To prepare the precursor solution, $0.36 \mathrm{~g}$ of glycine salt $\left(\mathrm{H}_{2} \mathrm{NCH}_{2} \mathrm{CO}_{2} \mathrm{H}\right.$, from Aldrich) was dissolved in $1.85 \mathrm{ml}$ of distilled water and $0.15 \mathrm{ml}$ of concentrated sulfuric acid $\left(\mathrm{H}_{2} \mathrm{SO}_{4}\right)$. The solution was warmed above room temperature $\left(\sim 40^{\circ} \mathrm{C}\right)$ and stirred for $24 \mathrm{~h}$. This saturated solution is usually used for obtaining TGS crystals when precipitation occurs. Prior to the electrospinning preparation, $0.83 \mathrm{~g}$ of PEO (Mw 100000 , from Aldrich) and $1.33 \mathrm{ml}$ of ethanol were added to the solution which was again stirred for $24 \mathrm{~h}$. Prepared in this way, the solution has a molar ratio of TGS/ethanol $=1.25$, which was found to be an optimum starting precursor ratio. Increasing the TGS part reduces the control of the electrospinning process. A further increase in the TGS concentration makes the nanofibre production impossible due to the rapid precipitation of TGS crystals in the precursor solution.

The prepared solution was loaded into a syringe with a metal needle of $0.8 \mathrm{~mm}$ inner diameter, which was connected to a $10-12 \mathrm{kV}$ dc voltage. The distance between the needle tip and the ground collector during electrospinning was varied in the range $12-15 \mathrm{~cm}$ and the fibres were spun at a flow rate of $1 \mathrm{ml} \mathrm{h}^{-1}$. The electrical potential, flow rate and the distance between the needle tip and the ground collector were adjusted in such a way as to obtain a stable jet. The specimens of the electrospun nanofibres were deposited on either an aluminium foil or optical glass.

The morphology and diameters of the electrospun fibres were determined by scanning electron microscopy (SEM). The SEM images were obtained with a Leica Cambridge S360 scanning electron microscope at $15 \mathrm{kV}$ and a working distance of $13 \mathrm{~mm}$. Samples were sputter coated with gold.

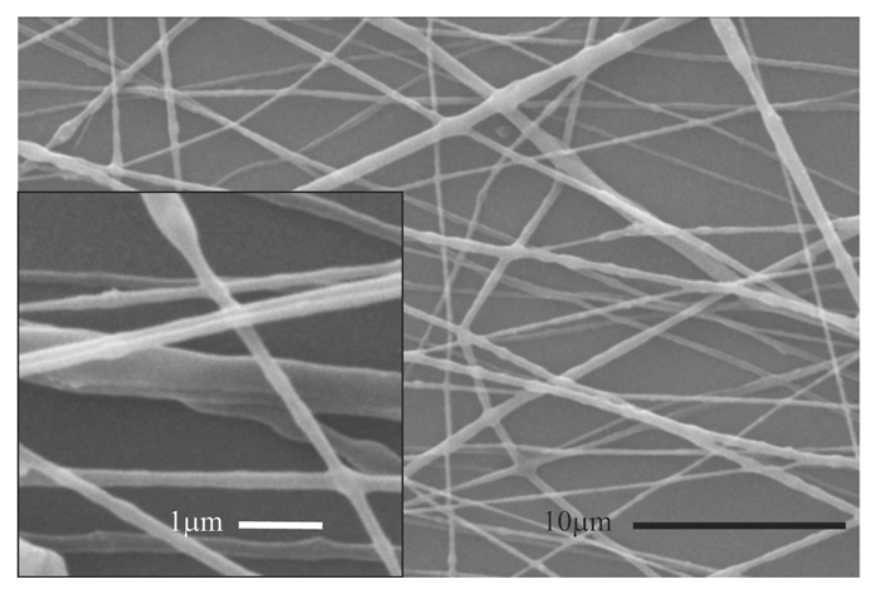

Figure 1. SEM micrograph of as-synthesized electrospun TGS-PEO fibres.

The crystal molecular structure was confirmed by Fourier transform (FT) IR spectroscopy with a Bruker IFS $66 \mathrm{~V}$ spectrometer. The polarized (transversal electric and transversal magnetic) attenuated total reflection (ATR) spectra were recorded from 500 to $4000 \mathrm{~cm}^{-1}$ with a resolution of $4 \mathrm{~cm}^{-1}$. For each spectra, 128 runs were collected and averaged. In a single reflection ATR system IR light enters at $45^{\circ}$ to the ATR crystal (diamond type IIa). The FT-IR spectra of the TGS-PEO nanofibres were measured on electrospun mats taken off from a glass or aluminium foil, where they were spread during the electrospinning process. The measurements were performed in reflectance mode with two polarizations.

The crystal distribution and domain structure of the TGS nanofibres were visualized using atomic force microscopy (AFM) with a conductive $\mathrm{Si}$ cantilever (Nanosensors) in the contact mode. The microscope (PicoPlus, Agilent Technologies) was equipped with an external lock-in amplifier (SR830, Stanford Research) and a function generator (FG120, Yokogawa), which were used to apply the ac and dc voltages to the fibre surface for poling and image acquisition [26]. The amplitude and frequency of the ac voltage were $1 \mathrm{~V}$ and $50 \mathrm{kHz}$, respectively. The fibre mat was deposited on an aluminium laminae to provide the conductive bottom electrode.

The temperature dependence of the dielectric permittivity was measured using a Wayne Kerr 6440A component analyzer at a constant heating rate of $1 \mathrm{~K} \mathrm{~min}^{-1}$ for different frequencies. Two parallel gold electrodes, with a length of $10 \mathrm{~mm}$ each and with a $1 \mathrm{~mm}$ gap between them, were deposited on the surface of a non-woven nanofibre mat. A sandwich-like electrode deposition procedure was carried out for the integral ferroelectric hysteresis measurements which were obtained by means of a standard Sawyer-Tower circuit with compensation of the dielectric loss.

\section{Results and discussion}

SEM micrographs obtained on an electrospun PEO-based TGS nanofibre mesh are shown in figure 1. The fibres have a smooth surface with non-uniform, beads-free, cylindrical parts with length exceeding $1 \mathrm{~cm}$ and average diameters ranging from 190 to $750 \mathrm{~nm}$. Such a wide diameter 


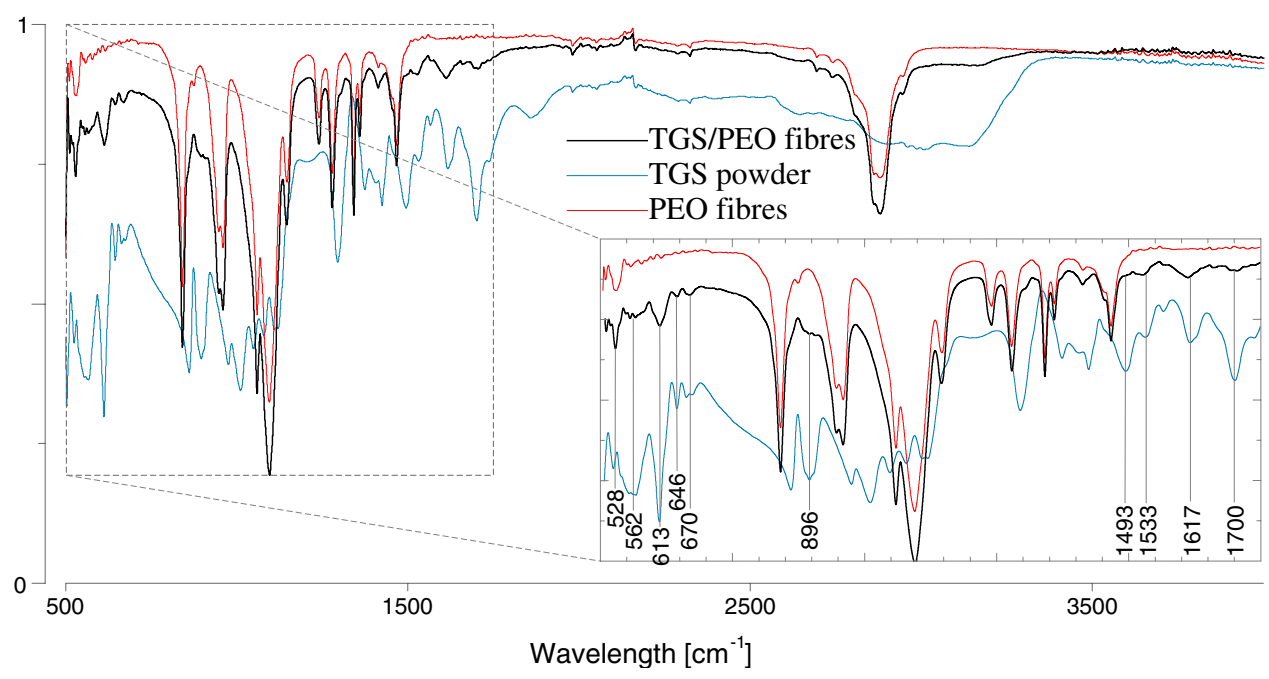

Figure 2. IR TE spectra obtained on TGS-PEO nanofibres (black curve) at room temperature. The spectra of a pure TGS crystal powder (blue, lower curve) and of PEO nanofibres (red, upper curve) are presented for comparison. The inset shows the expanded spectra for the range 500-1750 $\mathrm{cm}^{-1}$; vertical lines mark the frequencies of some modes of TGS that were obtained in TGS-PEO fibres.

distribution of the synthesized TGS-PEO fibres is due to the different electrical properties of the polymer and of the salt conglomeration, affecting the shape of the Taylor cone responsible for the stability of the electrospinning and spun fibres condition $[27,28]$.

Figure 2 shows the middle IR spectrum of an as-produced TGS-PEO nanofibre mesh. The spectra of a pure crystalline TGS powder sample and of pure PEO electrospun nanofibres are also presented in this figure. The observed IR peaks with their assignment are listed in table 1. There is clear evidence for the presence of the TGS crystals inside the PEO host. As can be seen, the peak positions of the TGS-PEO nanofibres spectrum, which can be related to the characteristic PEO or TGS peaks, remain unchanged. Some TGS peaks are partially overlapped with the more strong PEO bands. Thus, from the analysis of IR spectroscopic studies one can conclude that the spectrum of the TGS-PEO fibres is a superposition of the spectra of the two constituent phases (TGS and PEO) and there are no interactions of the TGS crystals with the PEO matrix.

Figure 3 presents the AFM topography and the domain structures of the individual TGS fibre obtained by piezoelectric response force microscopy (PFM) in the phase mode operation. The conventional PFM imaging is based on the detection of the mechanical response of the sample to an ac voltage applied (piezoresponse) via a conductive probing tip [29]. The linear coupling between the piezoelectric and ferroelectric constants implies that the domain polarity can be determined from the sign of the field-induced strain. In figure $3(b)$ an observed strong piezoelectric contrast, caused by additional deformation due to the applied low ac electric field, presents sub-micrometre ferroelectric domains allocated into the assynthesized unpoled TGS fibre. After applying a dc voltage of $+50 \mathrm{~V}$ for $10 \mathrm{~s}$ at the tip positioned at the side (marked by a cross) the dark area switched into a bright one (figure 3(c)). To induce an extra domain pattern of the opposite sign, the tip was moved to the other side of the fibre sample and an electric field of $-50 \mathrm{~V}$ was applied. A domain of the opposite sign, emerged in this way, which is presented in figure $3(d)$.
Table 1. IR bands observed in the range $500-4000 \mathrm{~cm}^{-1}$ for crystalline TGS powder, PEO fibre mat and TGS-PEO fibres (where $\delta$-bending, $\gamma$-rocking, $\nu$-stretching, $\omega$-wagging, $t$-twisting, $\tau$-torsion).

\begin{tabular}{llc}
\hline TGS $[30]$ & PEO [31] & TGS-PEO \\
\hline 524 & $530 v(\mathrm{COC})_{a s}(\mathrm{OCC})_{a s}(\mathrm{COC})$ & 528 \\
$562 \omega(\mathrm{CO})$ & & 562 \\
$613 v\left(\mathrm{SO}_{4}\right)$ & 613 \\
$646 \delta(\mathrm{COO})$ & & 646 \\
$670 \delta(\mathrm{COO})$ & $840 \gamma\left(\mathrm{CH}_{2}\right)_{a s}$ & 670 \\
& & 840 \\
$896 \gamma(\mathrm{CC})$ & $948 v\left(\mathrm{CH}_{2}\right)_{s}, v(\mathrm{COO}) \mathrm{a}$ & 896 \\
& 960 & 948 \\
& $1059 v\left(\mathrm{COC}_{s}, \tau\left(\mathrm{CH}_{2}\right)_{s}\right.$ & 960 \\
& $1095 v\left(\mathrm{CC}_{2}, \omega\left(\mathrm{CH}_{2}\right)_{s}\right.$ & 1059 \\
& $1240 t\left(\mathrm{CH}_{2}\right)_{s}$ & 960 \\
& $1279 t\left(\mathrm{CH}_{2}\right)_{a s}, t\left(\mathrm{CH}_{2}\right)_{s}$ & 1240 \\
$1295 \omega(\mathrm{CH})$ & 1340 & 1279 \\
& $1359 \omega\left(\mathrm{CH}_{2}\right) v(\mathrm{CC})$ & 1295 \\
& 1411 & 1340 \\
& $1465 \delta\left(\mathrm{CH}_{2}\right)_{a s}, \delta\left(\mathrm{CH}_{2}\right)_{s}$ & 1359 \\
$1493 \delta(\mathrm{CH})$ & & 1411 \\
$1533 \delta\left(\mathrm{NH}_{3}\right)$ & & 1465 \\
$1617 v(\mathrm{CO})_{s}$ & & 1533 \\
$1700 v(\mathrm{CO})$ & $2875 v\left(\mathrm{CH}_{2}\right)_{s}$ & 1617 \\
& & 1700 \\
& & 2875 \\
\hline
\end{tabular}

Since the PFM plays a crucial role in evaluating the ferroelectric nanostructures, the data obtained can be interpreted as follows. As shown in figure 4(a), due to the tendency of the TGS crystals to agglomerate the TGS/PEO volume fraction distribution along the fibre axis varies due to the attractive forces. During the electrospinning process, when the nucleation of the TGS crystals occurs, the zwitterion glycine molecule $\mathrm{NH}_{3}^{+} \mathrm{CH}_{2} \mathrm{COO}^{-}$(the other two molecules are protonated with $\mathrm{SO}_{4}^{2-}$ ) preferentially nucleates normal to the surface of the fibre due to the internal radial configuration 


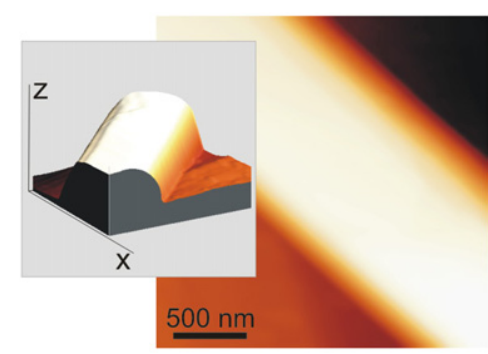

(a)

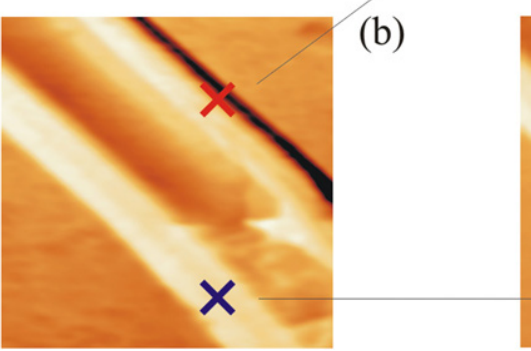

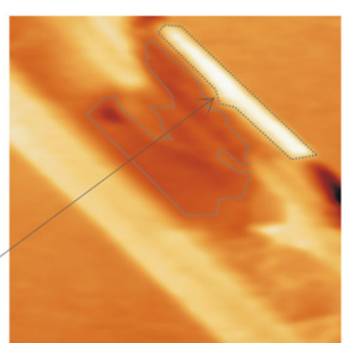

(c)

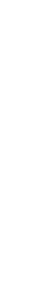

(d)

Figure 3. AFM and PFM images of an individual electrospun TGS fibre. (a) AFM topography and $(b)$ PFM image before poling. Crosses correspond to the tip positions where the dc electric field was applied. (c) PFM image obtained after poling at $+50 \mathrm{~V} ;(d)$ PFM image obtained after the next applied voltage at $-50 \mathrm{~V}$.

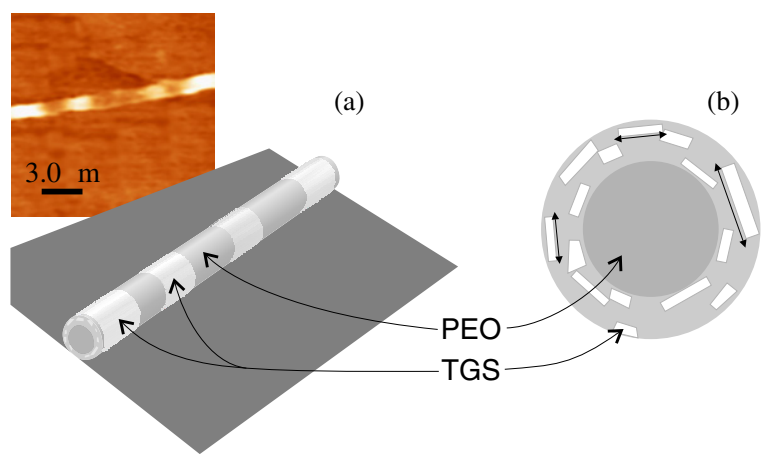

Figure 4. (a) PFM image and alteration of the TGS/PEO volume fraction along an individual TGS-PEO fibre axis; $(b)$ schematic cross section of the TGS-PEO fibre with dispersed crystals.

of the electrostatic field. Thus, the TGS crystals have a tendency to grow near the surface of the electrospun fibre with a two-fold axis in the plane parallel to the wall of the fibre, as shown by double arrows in figure $4(b)$. This assumption is confirmed by PFM imaging, since the domain structure of the fibres is determined solely by the TGS nanocrystals. A strong PFM contrast occurs at the borders of the fibre (bright area in figure $3(c)$ and dark one in figure $3(d)$ ) due to the fact that the polar axis of the nanocrystals is perpendicular to the surface normal of the fibre. A perpendicular direction of the polarization to the cantilever tip results in an absence of PFM contrast in the middle of the fibre (figures 3(b)- $(d)$ ).

In order to evaluate the integral ferroelectric properties of TGS fibres measurement of dielectric hysteresis loops was performed over a layer of fibre mat. For this, an ac electric field was applied to the electrospun sample deposited on an aluminium foil with an area of $0.25 \mathrm{~mm}^{2}$. A gold sheet was used as the upper electrode. Figure 5 presents the loops observed on the TGS nanofibres. Their shape, typical for

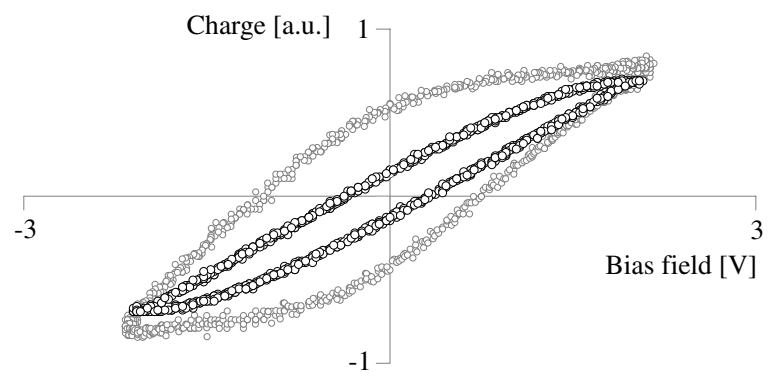

Figure 5. Lossy dielectric loops observed in TGS-PEO nanofibres.

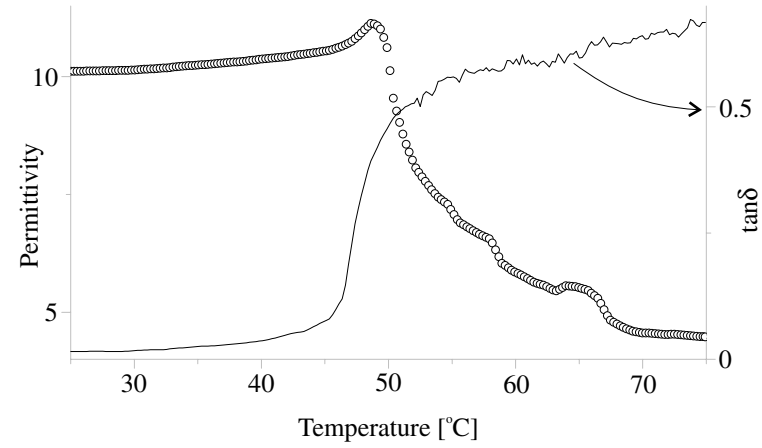

Figure 6. Temperature dependence of dielectric permittivity measured on the TGS-PEO nanofibres at $10 \mathrm{kHz}$.

lossy dielectrics, results from the contribution of the TGS nanocrystals and the polymer matrix.

The overall crystallinity and dielectric properties of the polymer-based TGS nanofibres were confirmed by measuring the temperature dependence of dielectric permittivity $\epsilon$. Figure 6 shows the dielectric permittivity $\epsilon$ and dielectric loss factor $\tan \delta$ as a function of temperature, measured at $10 \mathrm{kHz}$ on the TGS-PEO nanofibres. Initially, the electric permittivity slowly increases with increasing temperature up to about $49^{\circ} \mathrm{C}$ where it presents a maximum. Above this maximum $\epsilon(T)$ 
decreases sharply, presenting a broad peak at $65^{\circ} \mathrm{C}$ due to the melting of the polymer shell [32] on the nanofibres. On the other hand, the dielectric loss presents the opposite behaviour, having small values for temperatures below the maximum of the permittivity, increasing sharply in its vicinity and then increasing slowly above $55^{\circ} \mathrm{C}$. The maximum in $\epsilon(T)$ at $49^{\circ} \mathrm{C}$ is due to the ferroelectric-paraelectric phase transition characteristic of a TGS crystal. However, in contrast to the bulk crystalline TGS case, where its ferroelectric-paraelectric transition gives a sharp peak in $\epsilon(T)$ in the vicinity of the phase transition, in the nanofibres the corresponding peak is somewhat rounded and is smaller.

Such behaviour of the dielectric permittivity of the TGS nanofibres, namely the smooth and non-sharp peak in the vicinity of the phase transition, can be understood in terms of the two-phase effective medium model proposed by Bruggeman and further improved by Bergman [33]. When the composite is made of a sparse dispersion of spheres, with volume fraction $p_{1}$ and dielectric constant $\epsilon_{1}$, inside a homogeneous host medium with a dielectric constant $\epsilon_{2}$ and volume fraction $p_{2}$, the effective dielectric permittivity of the composite $\epsilon$ is obtained by $p_{1}\left(\epsilon_{1}-\epsilon\right) /\left(\epsilon_{1}+\epsilon\right)+p_{2}\left(\epsilon_{2}-\epsilon\right) /\left(\epsilon_{2}+\epsilon\right)=0$. Assuming that $p_{1}$ is much lower than $p_{2}$ the solution of this equation is reduced to $\epsilon \sim \sqrt{\epsilon_{1} \epsilon_{2}}$, showing a maximum at a temperature where either $\epsilon_{1}$ or $\epsilon_{2}$ attains a peak, as in the case of TGS near the phase transition temperature.

\section{Conclusion}

Nanofibres of semiorganic TGS have been synthesized from a polymer solution of PEO using the electrospinning method. The FT-IR spectra measured in electrospun nanofibres confirmed the presence of TGS crystals dispersed in the PEO matrix. Switchable ferroelectric domains of the TGS particles were observed along the fibres by the use of PFM. The simple interpretation where the TGS crystals have preferable nucleation on the fibre periphery due to an internal radial electrostatic field causing glycine dipole orientation was discussed. A phase transition on the dielectric permittivity was observed in the vicinity of $49^{\circ} \mathrm{C}$, which was due to the ferroelectric-paraelectric phase transformation of TGS.

The significant piezoelectric response obtained on TGSPEO fibres in combination with the cost-effective synthesizing method of electrospinning makes this approach very attractive for producing semiorganic nanofibre arrays with enhanced properties.

\section{Acknowledgments}

This work was financially supported by Fundacao para a Ciencia e Tecnologia (reference CIENCIA-2007 UMINHOCF-06). The authors would like to acknowledge Luis Vieira for help in FT-IR measurements.

\section{References}

[1] Li D, McCann J T and Xia Y N 2006 J. Am. Ceram. Soc. 891861

[2] Subbiah T, Bhat G S, Tock R W, Parameswaran S and Ramkumar S S 2005 J. Appl. Polym. Sci. 96557

[3] Martins A, Araujo J V, Reis R L and Neves N M 2007 Nanomedicine 2929

[4] Smith L A and Ma P X 2004 Colloids Surf. B: Biointerfaces 39125

[5] Katti D S, Robinson K W, Ko F K and Laurencin C T 2004 J. Biomed. Mater. Res. B 70286

[6] Schiffman J D and Schauer C L 2008 Polym. Rev. 48317

[7] Nair L S, Bhattacharyya S, Bender J D, Greish Y E, Brown P W, Allcock H R and Laurencin C T 2004 Biomacromolecules 5 2212-20

[8] Barhate R S and Ramakrishna S J 2007 Membrane Sci. 2961

[9] Larsen G, Velarde-Ortiz R, Minchow K, Barrero A and Loscertales I G 2003 J. Am. Chem. Soc. 1251154

[10] Berutti F A, Alves A K, Bergmann C P, Clemens F J and Graule T 2009 Particulate Sci. Technol. 27203

[11] Bognitzki M, Hou H, Ishaque M, Frese T, Hellwig M, Schwarte C, Shaper A, Wendorff J H and Greiner A 2000 Adv. Mater. 12637

[12] Shao C, Kim H Y, Gong J, Ding B, Lee D R and Park S J 2003 Mater. Lett. 571579

[13] Li D, Wang Y L and Xia Y N 2003 Nano Lett. 31167

[14] Ahn C H, Rabe K M and Triscone J M 2004 Science 303 488-91

[15] Dawber M, Rabe K M and Scott J F 2005 Rev. Mod. Phys. 77 1083-130

[16] Ziebert C, Schmitt H, Krüger J K, Sternberg A and Ehses K H 2004 Phys. Rev. B 69214106

[17] Khutorsky V E and Lang S B 1997 J. Appl. Phys. 82 1288-92

[18] Yang Y, Chan H L W and Choy C L 2006 J. Mater. Sci. 41 251-8

[19] Nitzani M and Berger S 2007 Physica E 37260

[20] Bootsma G A and Gassen H J 1971 J. Cryst. Growth $10223-7$

[21] Wang N, Tang Y H, Zhang Y F, Lee C S and Lee S T 1998 Phys. Rev. B 58 R 16024

[22] Choi S J and Park S M 2000 Adv. Mater. 12 1547-49

[23] Wu C G and Bein T 1994 Science 264 1757-59

[24] Reneker D H, Yarin A L, Fong H and Koombhongse S $2000 \mathrm{~J}$. Appl. Phys. 87 4531-47

[25] Theron S A, Zussman E and Yarin A L 2004 Polymer 45 2017-30

[26] Kholkin A L, Bdikin I K, Shvartsman V V and Pertsev N A 2007 Nanotechnology 18095502

[27] Friedreich S V, Yu J H, Brenner M P and Rutledge G C 2003 Phys. Rev. Lett. 90144502

[28] Samatham R and Kim K J 2006 Polym. Eng. Sci. 46 954-59

[29] Christman J A, Woolcott R R Jr, Kingon A I and Nemanicha R J 1998 Appl. Phys. Lett. 73 3851-3

[30] Winterfeldt V, Schaak G and Klopperpieper A 1977 Ferroelectrics $\mathbf{1 5} 21$

[31] Yoshihara T, Tadokoro H and Murahash S 1964 J. Chem. Phys. 412902

[32] Ratna D, Abraham T N and Karger-Kocsis J 2008 J. Appl. Polym. Sci. 108215662

[33] Bergman D J 1978 Phys. Rep. 43377 